Homology, Homotopy and Applications, vol.20(2), 2018, pp.105-114

\title{
PARTIAL EULER CHARACTERISTIC, NORMAL GENERATIONS AND THE STABLE $D(2)$ PROBLEM
}

\author{
FENG JI AND SHENGKUI YE
}

(communicated by J.P.C. Greenlees)

\begin{abstract}
We study the interplay among Wall's $D(2)$ problem, the normal generation conjecture (the Wiegold Conjecture) of perfect groups and Swan's problem on partial Euler characteristic and deficiency of groups. In particular, for a 3-dimensional complex $X$ of cohomological dimension 2 with finite fundamental group, assuming the Wiegold conjecture holds, we prove that $X$ is homotopy equivalent to a finite 2-complex after wedging a copy of sphere $S^{2}$.
\end{abstract}

\section{Introduction}

In this article, we study several classical problems in low-dimensional homotopy theory and group theory, focusing on the interplay among these problems.

Let us first recall Swan's problem. Let $G$ be a group and $\mathbb{Z} G$ the group ring. Swan [16] defines the partial Euler characteristic $\mu_{n}(G)$ as follows. Let $F$ be a resolution

$$
\cdots \rightarrow F_{2} \rightarrow F_{1} \rightarrow F_{0} \rightarrow \mathbb{Z} \rightarrow 0
$$

of the trivial $\mathbb{Z} G$-module $\mathbb{Z}$, in which each $F_{i}$ is $\mathbb{Z} G$-free on $f_{i}$ generators. For an integer $n \geqslant 0$, if

$$
f_{0}, f_{1}, f_{2}, \ldots, f_{n}
$$

are finite, define

$$
\mu_{n}(F)=f_{n}-f_{n-1}+f_{n-2}-\cdots+(-1)^{n} f_{0} .
$$

If there exists a resolution $F$ such that $\mu_{n}(F)$ is defined, we let $\mu_{n}(G)$ be the infimum of $\mu_{n}(F)$ over all such resolutions $F$. We call the truncated free resolution

$$
F_{n} \rightarrow \cdots \rightarrow F_{1} \rightarrow F_{0} \rightarrow \mathbb{Z} \rightarrow 0
$$

an algebraic $n$-complex if each $F_{i}$ is finitely generated as a $\mathbb{Z} G$-module (following the terminology of Johnson $[\mathbf{8}]$ ).

For a finitely presentable group $G$, the deficiency $\operatorname{def}(G)$ is the maximum of $d-k$ over all presentations $\left\langle g_{1}, g_{2}, \ldots, g_{d} \mid r_{1}, r_{2}, \ldots, r_{k}\right\rangle$ of $G$. It is not hard to see that

$$
\operatorname{def}(G) \leqslant 1-\mu_{2}(G)
$$

$[\mathbf{1 6}$, Proposition 1]. However, Swan mentions in [16] that "the problem of determining

Received November 3, 2017, revised December 28, 2017; published on May 9, 2018.

2010 Mathematics Subject Classification: 57M20, 57M05.

Key words and phrases: D(2) problem, cohomological dimensions, Quillen's plus construction.

Article available at http://dx.doi.org/10.4310/HHA.2018.v20.n2.a6

Copyright (C) 2018, International Press. Permission to copy for private use granted. 
when $\operatorname{def}(G)=1-\mu_{2}(G)$ seems very difficult even if $G$ is a finite $p$-group".

Next, we consider Wall's $D(2)$ problem $(c f$. [17]). The cohomological dimension $\operatorname{cd}(X)$ of a CW complex $X$ is defined as the largest integer $n$ such that $H^{n}(X, M) \neq 0$ for some $\mathbb{Z}\left[\pi_{1}(X)\right]$-module $M$. For a 3-dimensional CW complex $X$ of cohomological dimension $\operatorname{cd}(X)=2$, Wall's $D(2)$ problem asks whether $X$ is homotopy equivalent to a 2-dimensional CW complex. A positive answer to this problem will imply the Eilenberg-Ganea conjecture, which says that a group of cohomological dimension two has a 2-dimensional classifying space. A finitely presentable group $G$ is said to have $D(2)$ property if any finite 3-dimensional CW complex $X$, of cohomological dimension 2 with fundamental group $G$, is homotopy equivalent to a 2-dimensional CW complex. For the status of $D(2)$ problem, see Johnson $[\mathbf{8}, \mathbf{9}]$ (see also $[\mathbf{5}, \mathbf{7}]$ for some recent work).

It is well-known that a finite perfect group $G$ is normally generated by one element $[\mathbf{1 1}, 4.2]$. The Wiegold conjecture ( $c f .[\mathbf{1}, \mathrm{FP} 14]$ and $[\mathbf{1 5}, 5.52])$ asserts that the same holds for any finitely generated perfect group:

Conjecture 1.1 (Wiegold conjecture). Let $G$ be any finitely generated perfect group, i.e. $G=[G, G]$, the commutator subgroup of $G$. Then $G$ can be normally generated by a single element.

Our main result is the following, which gives a relaxed lower bound of $\operatorname{def}(G)$ assuming the Wiegold conjecture.

Theorem 1.2. Assume that Conjecture 1.1 is true. Let $X$ be a finite 3-dimensional $C W$ complex of cohomological dimension 2 with finite fundamental group. We have the following:

(i) the complex $X$ is homotopy equivalent to a finite 3-dimensional complex with just one 3-cell;

(ii) the wedge $X \vee S^{2}$ is homotopy equivalent to a finite 2-dimensional complex;

(iii) $1-\mu_{2}(G) \geqslant \operatorname{def}(G) \geqslant-\mu_{2}(G)$ for any finite group $G$.

Our discussions are based on the study of a stable version of the $D(2)$ problem (for details, see Section 3). For a group $G$ having a finite classifying space B $G$ of dimension at most 2 , we have $\operatorname{def}(G)=1-\mu_{2}(G)$, which confirms the equality of partial Euler characteristic and deficiency (cf. Theorem 4.3 (i)). A famous conjecture of Whitehead says that any subcomplex of an aspherical 2-dimensional CW complex is aspherical $(c f .[\mathbf{2}])$. As an application of the results proved, we reprove the following (cf. Bogley [2]).

Corollary 1.3. A subcomplex $X$ of a finite aspherical 2-dimensional $C W$ complex is aspherical if and only if the fundamental group $\pi_{1}(X)$ has a finite classifying space $\mathrm{B} \pi_{1}(X)$ of dimension at most 2 .

The article is organized as follows. In Section 2, we discuss the Quillen plus construction of 2-dimensional CW complexes. This motivates the stable Wall's $D(2)$ property being discussed in Section 3. In the last section, the Euler characteristics are studied for groups of low geometric dimensions. 


\section{Quillen's plus construction of 2-dimensional CW complexes}

Let $X$ be a CW complex with fundamental group $G$ and $P$ a perfect normal subgroup of $G$, i.e. $P=[P, P]$. Quillen shows that there exists a CW complex $X_{P}^{+}$, whose fundamental group is $G / P$; and an inclusion $f: X \rightarrow X_{P}^{+}$such that

$$
H_{n}\left(X ; f^{*} M\right) \cong H_{n}\left(X_{P}^{+} ; M\right),
$$

for any integer $n$ and local coefficient system $M$ over $X_{P}^{+}$. Here $X_{P}^{+}$is called the plusconstruction of $X$ with respect to $P$. It is unique up to homotopy equivalence. One of the main applications of the plus construction is to define higher algebraic $K$-theory. In general, the space $X_{P}^{+}$is obtained from $X$ by attaching 2-cells and 3-cells. We need the following definition.

Definition 2.1. The cohomological dimension $\operatorname{cd}(X)$ of a CW complex $X$ is defined as the smallest integer $n$ such that $H^{m}(X, M)=0$ for any integer $m>n$ and any $\mathbb{Z}\left[\pi_{1}(X)\right]$-module $M$. If no such $n$ exists, the cohomological dimension $\operatorname{cd}(X)$ is defined to be $\infty$.

It is obvious that an $n$-dimensional CW complex is of cohomological dimension at most $n$. The following well-known lemma gives a property enjoyed by any 3dimensional CW complex with cohomological dimension 2.

Lemma 2.2. Suppose that $X$ is a 3-dimensional $C W$ complex and $\tilde{X}$ is the universal cover of $X$. Let $C_{*}(\tilde{X})$ be the cellular chain complex of $\tilde{X}$. Then $X$ is of cohomological dimension 2 if and only if the image of $C_{3}(\tilde{X})$ is a direct summand of $C_{2}(\tilde{X})$ as $\mathbb{Z}\left[\pi_{1}(X)\right]$-modules.

The following result shows that for certain 2-dimensional CW complexes, the Quillen plus construction is homotopy equivalent to a 2-dimensional CW complex. Let $X$ be a finite 2-dimensional CW complex. Suppose that a perfect normal subgroup $P$ in $\pi_{1}(X)$ is normally generated by $n$ elements. With respect these normal generators, there is a canonical construction $Y$ for $X^{+}$that attaches a 2-cell bounded by each generator and a 3-cell to kill the resulting homology. Moreover, the number of attached 3-cells and the number of attached 2-cells are both $n$ ( $c f$. the proof of Theorem 1 in $[\mathbf{1 8}])$. The cellular chain complex $C_{*}(\tilde{Y})$ of the universal cover $\tilde{Y}$ is

$$
\begin{aligned}
0 \rightarrow \mathbb{Z}\left[\pi_{1}(Y)\right]^{n} \rightarrow \mathbb{Z}[ & \left.\pi_{1}(Y)\right]^{n} \oplus C_{2}(\tilde{X}) \otimes_{\mathbb{Z}\left[\pi_{1}(X)\right]} \mathbb{Z}\left[\pi_{1}(Y)\right] \\
& \rightarrow C_{1}(\tilde{X}) \otimes_{\mathbb{Z}\left[\pi_{1}(X)\right]} \mathbb{Z}\left[\pi_{1}(Y)\right] \rightarrow C_{0}(\tilde{X}) \otimes_{\mathbb{Z}\left[\pi_{1}(X)\right]} \mathbb{Z}\left[\pi_{1}(Y)\right] \rightarrow 0,
\end{aligned}
$$

with the first map the inclusion of a direct summand. This is homotopy equivalent to

$$
\begin{aligned}
0 \rightarrow C_{2}(\tilde{X}) \otimes_{\mathbb{Z}\left[\pi_{1}(X)\right]} \mathbb{Z}\left[\pi_{1}(Y)\right] \rightarrow C_{1}(\tilde{X}) \otimes_{\mathbb{Z}\left[\pi_{1}(X)\right]} & \mathbb{Z}\left[\pi_{1}(Y)\right] \\
& \rightarrow C_{0}(\tilde{X}) \otimes_{\mathbb{Z}\left[\pi_{1}(X)\right]} \mathbb{Z}\left[\pi_{1}(Y)\right] \rightarrow 0 .
\end{aligned}
$$

It follows that

Lemma 2.3. The plus construction $\left(X \vee\left(S^{2}\right)^{\vee n}\right)^{+}$of the wedge of $X$ and $n$ copies of $S^{2}$, taken with respect to $P$, is homotopy equivalent to the 2-skeleton of $Y$.

The following lemma is from Johnson $[8,59.4$, p. 228]. Although the original version is stated for complexes with finite groups, it does hold for complexes with finitely presentable groups ( $c f$. [8, appendix B] and Mannan [14]). 
Lemma 2.4. Let $Y$ be a finite 3-dimensional $C W$ complex of cohomological dimension 2. If the reduced chain complex of the universal cover

$$
0 \rightarrow C_{2}(\tilde{Y}) / C_{3}(\tilde{Y}) \rightarrow C_{1}(\tilde{Y}) \rightarrow \mathbb{Z} \pi_{1}(Y) \rightarrow \mathbb{Z} \rightarrow 0
$$

is homotopy equivalent to the chain complex of the universal cover of a 2-dimensional $C W$ complex $X$, then $Y$ is homotopy equivalent to $X$.

\section{Wall's $\mathrm{D}(2)$ problem and its stable version}

In this section, we apply the results obtained in the previous section to study the $D(2)$ problem. Let us recall the $D(2)$ problem raised in [17].

Conjecture 3.1 (The $D(2)$ problem). If $X$ is a finite 3-dimensional $C W$ complex of cohomological dimension at most 2 , then $X$ is homotopy equivalent to a 2-dimensional CW complex.

In $[\mathbf{8}]$, Johnson proposes to systematically study the problem by parameterizing 3 -dimensional CW complexes by their fundamental groups. For a finitely presentable group $G$, we say the $D(2)$ problem is true for $G$, if any finite 3 -dimensional CW complex $X$, of cohomological dimension at most 2 with fundamental group $\pi_{1}(X)=$ $G$, is homotopy equivalent to a 2 -dimensional $\mathrm{CW}$ complex.

The $D(2)$ problem is very difficult in general. It is known to be true for a limited amount of groups (for an updated state, see $[\mathbf{4}, \mathbf{1 2}]$ and $[\mathbf{9}$, p. 261]). We propose the following stable version by allowing taking wedge with copies of $S^{2}$.

Conjecture 3.2 (The $D(2, n)$ problem). Let $n \geqslant 0$ be an integer. If $X$ is a finite 3-dimensional $C W$ complex of cohomological dimension at most 2 , then $X \vee\left(S^{2}\right)^{\vee n}$ is homotopy equivalent to a 2-dimensional CW complex.

For a finitely presentable group $G$ and an integer $n \geqslant 0$, we say that $G$ has the $D(2, n)$ property (or the $D(2, n)$ problem holds for $G$ ) if Conjecture 3.2 is true for all those $X$ with fundamental group $G$. The $D(2,0)$ problem is the original $D(2)$ problem. It is immediate that property $D(2)$ implies $D(2, n)$; and $D(2, n)$ implies $D(2, n+1)$ for any group $G$ and any integer $n \geqslant 0$.

We now study the relation between the stabilization by wedging copies of $S^{2}$ with that by attaching 3-cells.

Proposition 3.3. Suppose that $X$ is a finite 3-dimensional $C W$ complex of cohomological dimension at most 2. Then $X \vee\left(S^{2}\right)^{\vee n}$ is homotopy equivalent to a finite 2-dimensional $C W$ complex if and only if $X$ is homotopy equivalent to a 3-dimensional CW complex with $n$ 3-cells.

Proof. Assume that $X$ is homotopy equivalent to a 3-dimensional CW complex $X^{\prime}$ with $n$ 3-cells. Denote by $X^{\prime(2)}$ the 2 -skeleton of $X^{\prime}$ and let $Z=X^{\prime} \vee\left(S^{2}\right)^{n}$. It is not hard to see that the reduced chain complex

$$
0 \rightarrow C_{2}(\tilde{Z}) / C_{3}(\tilde{Z}) \rightarrow C_{1}(\tilde{Z}) \rightarrow \mathbb{Z} \pi_{1}(Z) \rightarrow \mathbb{Z} \rightarrow 0
$$

is homotopy equivalent to the chain complex of the universal cover of $X^{\prime(2)}$. By Lemma 2.4, $X \vee\left(S^{2}\right)^{\vee n}$ is homotopy equivalent to a 2-dimensional CW complex.

Conversely, suppose that $X \vee\left(S^{2}\right)^{\vee n}$ is homotopy equivalent to a finite 2-complex $Y$ via a map $f: X \vee\left(S^{2}\right)^{\vee n} \rightarrow Y$. It is clear that 


$$
\pi_{1}(X)=\pi_{1}\left(X \vee\left(S^{2}\right)^{\vee n}\right) \cong \pi_{1}(Y) .
$$

Let $G=\pi_{1}(X)$ and $\tilde{X}, \tilde{Y}$ be the universal covering spaces of $X, Y$ respectively. By the Hurewicz theorem, we have isomorphisms

$$
\pi_{2}(Y) \cong \pi_{2}(\tilde{Y}) \cong H_{2}(\tilde{Y}) \cong \pi_{2}(\tilde{X}) \oplus \mathbb{Z} G^{n} .
$$

Therefore, there are $n$ maps $f_{i}: S^{2} \rightarrow Y, 1 \leqslant i \leqslant n$, corresponding to the inclusion onto the second factor (for a fixed basis of $\mathbb{Z} G^{n}$ )

$$
\mathbb{Z} G^{n} \rightarrow H_{2}(\tilde{Y}) \cong \pi_{2}(\tilde{X}) \oplus \mathbb{Z} G^{n} .
$$

Attaching 3-cells to $Y$ along these $f_{i}(1 \leqslant i \leqslant n)$, we obtain a 3 -dimensional CW complex $Y \cup_{i=1}^{n} e_{i}^{3}$. Let $i: X \stackrel{i}{\rightarrow} X \vee\left(S^{2}\right)^{\vee n}$ be the natural inclusion. By our construction, the canonical composition

$$
f^{\prime}: X \stackrel{i}{\rightarrow} X \vee\left(S^{2}\right)^{\vee n} \stackrel{f}{\rightarrow} Y \rightarrow Y \cup_{i=1}^{n} e_{i}^{3}
$$

induces isomorphisms on both $\pi_{1}$ and $\pi_{2}$ (the same as the second homology groups of the universal covers). It is not hard to see that

$$
H_{3}(\tilde{X})=H_{3}\left(Y \widetilde{\cup_{i=1}^{n}} e_{i}^{3}\right)=0 .
$$

Therefore, $f^{\prime}$ induces a homotopy equivalence between the chain complexes of the universal covering spaces. By the Whitehead theorem, $f^{\prime}$ is a homotopy equivalence.

Proof of Theorem 1.2 (i) and (ii). By Proposition 3.3, (i) is equivalent to (ii). We prove (ii) as follows. By a result of Mannan [13], $X$ is the plus construction of a finite 2-complex $Y$ with respect to a perfect normal subgroup $P \leqslant \pi_{1}(Y)$. Therefore, we have a short exact sequence of groups

$$
1 \rightarrow P \rightarrow \pi_{1}(Y) \rightarrow \pi_{1}(X) \rightarrow 1
$$

Since $\pi_{1}(Y) / P=\pi_{1}(X)$ is finite and $Y$ is finite, the covering space of $Y$ with fundamental group $P$ is again a finite CW complex. Hence $P$ is finitely generated. If the normal generation conjecture (Conjecture 1.1) holds, $P$ is normally generated by a single element. Lemma 2.3 says that $X \vee S^{2}$ is homotopy equivalent to a 2 dimensional CW complex.

Without the assumption of the Wiegold conjecture we only know that a finite group $G$ has property $D(2, n)$ for $n=\max \left\{1,1-\operatorname{def}(G)-\mu_{2}(G)\right\}$, which follows the Swan-Jacobinski theorem in [8, 29.3, 29.4] and Browning's results [3].

\section{Partial Euler characteristic and the Whitehead conjecture}

Recall definitions of $\mu_{n}(F)$ for an algebraic $n$-complex $F_{*}$ and $\mu_{n}(G)$ from Introduction. For a finitely presentable group $G$, the following lemma follows from Swan [16] easily.

Lemma 4.1. Assume that $G$ is finitely presentable. The invariant $\mu_{2}(G)$ can be realized by an algebraic 2-complex. In other words, there exists an algebraic 2-complex

$$
F_{2} \rightarrow F_{1} \rightarrow F_{0} \rightarrow \mathbb{Z} \rightarrow 0
$$


such that

$$
\mu_{2}(G)=\operatorname{dim}_{\mathbb{Z} G} F_{2}+\operatorname{dim}_{\mathbb{Z} G} F_{0}-\operatorname{dim}_{\mathbb{Z} G} F_{1} .
$$

Proof. It is enough to notice that $\mu_{2}(G)$ is finite by Theorem 1.2 in [16].

Proof of Theorem 1.2 (iii). We prove a more general result: if a finitely presentable group $G$ satisfies the $D(2, n)$ problem, then

$$
\operatorname{def}(G) \geqslant(1-n)-\mu_{2}(G) .
$$

By Lemma 4.1, we can choose an algebraic 2-complex

$$
\left(F_{*}\right): F_{2} \rightarrow F_{1} \rightarrow F_{0} \rightarrow \mathbb{Z} \rightarrow 0
$$

such that

$$
\mu_{2}(G)=\operatorname{dim}_{\mathbb{Z} G} F_{2}+\operatorname{dim}_{\mathbb{Z} G} F_{0}-\operatorname{dim}_{\mathbb{Z} G} F_{1} .
$$

Since every algebraic 2-complex is geometric realizable by a 3 -dimensional $\mathrm{CW}$ complex (cf. Johnson [8, Theorem 60.2]), there is a finite 3-dimensional CW complex of cohomological dimension 2 such that the reduced chain complex

$$
C_{2}(\tilde{Y}) / C_{3}(\tilde{Y}) \rightarrow C_{1}(\tilde{Y}) \rightarrow \mathbb{Z} \pi_{1}(Y) \rightarrow \mathbb{Z} \rightarrow 0
$$

is homotopy equivalent to $\left(F_{*}\right)$. Assuming that $G$ has the $D(2, n)$ property, the wedge $X \vee\left(S^{2}\right)^{\vee n}$ is homotopy equivalent to a 2-dimensional CW complex, which gives a presentation of $G$. This implies that $\mu_{2}(G)+n \geqslant 1-\operatorname{def}(G)$, i.e. $\operatorname{def}(G) \geqslant(1-n)-$ $\mu_{2}(G)$. When Wiegold's Conjecture holds, the complex $X$ has property $D(2,1)$, which gives (iii).

It is possible to place $\mu_{2}(G)$ in the broader setting of $(G, n)$-complexes, as follows (cf. [6]). Recall that a $(G, n)$-complex is a finite $n$-dimensional CW complex $X$ with fundamental group $G$ and vanishing homotopy group $\pi_{i}(X)=0$ for $i=2,3, \ldots, n-1$. In particular, a $(G, 2)$-complex is a usual finite 2-dimensional $\mathrm{CW}$ complex with fundamental group $G$.

Definition 4.2. Let $G$ be a finitely presentable group. Define

$$
\mu_{n}^{g}(G)=\min \left\{(-1)^{n} \chi(X) \mid X \text { is a }(G, n) \text {-complex }\right\} .
$$

If there is no such $X$ exists, define $\mu_{n}^{g}(G)=+\infty$. We call that a $(G, n)$-complex $X$ with $(-1)^{n} \chi(X)=\mu_{n}^{g}(G)$ is a complex realizing $\mu_{n}^{g}(G)$.

A few observations are immediate. It is clearly true that $\mu_{n}(G) \leqslant \mu_{n}^{g}(G)$. Therefore, $\mu_{n}^{g}(G)>-\infty$ since $\mu_{n}(G)>-\infty$ (cf. Swan $\left.[\mathbf{1 6}]\right)$. Moreover, $\mu_{2}(G)=\mu_{2}^{g}(G)$ if and only if $\mu_{2}(G)=1-\operatorname{def}(G)$.

Now we study the partial Euler characteristic and deficiency for groups of low geometric dimensions. Recall that for a group $G$, the classifying space B $G$ of $G$ is defined as the connected CW complex with $\pi_{1}(\mathrm{~B} G)=G$ and $\pi_{i}(\mathrm{~B} G)=0, i \geqslant 2$. It is unique up to homotopy.

Theorem 4.3. Let $G$ be a group having a finite $n$-dimensional classifying space $\mathrm{B} G$. We have the following: 
(i) $\mu_{n}(G)=\mu_{n}^{g}(G)$; In particular, $\mu_{2}(G)=1-\operatorname{def}(G)$ if $G$ has a finite 2-dimensional $\mathrm{B} G$;

(ii) Any finite $C W$ complex $X$ with $\pi_{1}(X)=G$ satisfying the following properties:

a) the dimension is at most $n+1$;

b) the cohomological dimension $\operatorname{cd}(X)$ is at most $n$;

c) if $n \geqslant 3$, the homotopy group $\pi_{i}(X)=0$ for $2 \leqslant i \leqslant n-1$;

d) $(-1)^{n} \chi(X)=\mu_{n}^{g}(G)$,

is homotopy equivalent to $\mathrm{B} G$.

Proof. Let EG be the universal cover of $\mathrm{B} G$. Since $\mathrm{E} G$ is contractible, one obtains the exact cellular chain complex of $\mathrm{E} G$ :

$$
C_{*}(\mathrm{E} G): 0 \rightarrow C_{n}(\mathrm{E} G) \rightarrow C_{n-1}(\mathrm{E} G) \rightarrow \cdots \rightarrow \mathbb{Z} G \rightarrow 0 .
$$

This gives a (truncated) free resolution of $G$. In order to prove (i), it suffices to show that this resolution gives the minimal Euler characteristic $\mu_{n}(G)$ since we notice earlier that $\mu_{n}(G) \leqslant \mu_{n}^{g}(G)$.

Suppose that $\mu_{n}(G)$ is obtained from the following partial resolution of finitely generated free $\mathbb{Z} G$-modules:

$$
F_{*}: F_{n} \stackrel{d}{\rightarrow} F_{n-1} \rightarrow \cdots \rightarrow F_{1} \rightarrow \mathbb{Z} G \rightarrow 0 .
$$

We claim that $F_{*}$ is exact at $F_{n}$, i.e. $\operatorname{ker} d=0$. Once this is proved, $C_{*}(\mathrm{E} G)$ and $F_{*}$ are chain homotopic to each other and hence have the same Euler characteristic.

To prove the claim, let $J$ be the kernel of $d$. By Schanuel's lemma, there is an isomorphism

$$
J \oplus C_{n}(\mathrm{E} G) \oplus F_{n-1} \oplus \cdots \cong F_{n} \oplus C_{n-1}(\mathrm{E} G) \oplus \cdots .
$$

Applying the functor $-\otimes_{\mathbb{Z} G} \mathbb{Z}$ to both sides of this isomorphism, we see that $\mu_{n}(F)=$ $(-1)^{n} \chi(\mathrm{B} G)$ and $J \otimes_{\mathbb{Z} G} \mathbb{Z}=0$ by noticing the fact that the complex $F_{*}$ attains minimal Euler characteristic after multiplying $(-1)^{n}$ among all the algebraic $n$-complexes. This implies that $C_{n}(\mathrm{E} G) \oplus F_{n-1} \oplus \cdots$ and $F_{n} \oplus C_{n-1}(\mathrm{E} G) \oplus \cdots$ have the same finite free $\mathbb{Z} G$-rank. By Kaplansky's theorem, $J$ is the trivial $\mathbb{Z} G$-module(cf. [10], p. 328). This proves (i).

We now prove (ii). Let $C_{*}(\tilde{X})$ be the chain complex of the universal covering space of $X$. Since $\operatorname{cd}(X) \leqslant n, C_{n+1}(\tilde{X})$ is a direct summand of $C_{n}(\tilde{X})$, by the same argument given in Lemma 2.2. Let $F^{1}$ be the chain complex

$$
F_{*}^{1}: C_{n}(\tilde{X}) / C_{n+1}(\tilde{X}) \stackrel{d}{\rightarrow} C_{n-1}(\tilde{X}) \rightarrow \cdots \rightarrow C_{1}(\tilde{X}) \rightarrow \mathbb{Z} G \rightarrow 0 .
$$

It is not hard to see that $\pi_{n}(X) \cong \operatorname{ker} d$. Note that

$$
\mu_{n}\left(F^{1}\right)=(-1)^{n} \chi(X)=\mu_{n}(G) .
$$

By the same argument as the first part of the proof, we get ker $d=0$. This implies that $\tilde{X}$ is $n$-connected. Since $H_{n+1}(\tilde{X})=0, \tilde{X}$ is contractible and $X$ is homotopy equivalent to $\mathrm{B} G$.

Remark 4.4. Under the condition of Theorem 4.3, Harlander and Jensen [6] already prove that a $(G, n)$-complex realizing $\mu_{n}^{g}(G)$ is homotopy equivalent to $\mathrm{B} G$. Note that a $(G, n)$-complex is a special case of $X$ in Theorem 4.3 . 
We conclude with an application. Suppose that $G$ is a finitely presentable group and

$$
\mathbf{P}=\left\langle x_{1}, \ldots, x_{n} \mid r_{1}, \ldots, r_{m}\right\rangle
$$

is a presentation of $G$. Denote by $G_{\mathbf{P}}$ the group given by the presentation $\mathbf{P}$. From each finite 2-dimensional $\mathrm{CW}$ complex $X$, one shrinks a spanning tree in the 1-skeleton to make $X$ have only a single 0 -cell and obtains a finite presentation of $\pi_{1}(X)$. Namely, the 1-cells correspond one-one to a set of generators while the 2-cells correspond oneone to a set of relators. Therefore, any counter-example to the Whitehead conjecture gives rise to a 2-complex with a single 0 -cell. For a presentation $\mathbf{P}$, we will denote by $\chi(\mathbf{P})=m-n+1$. A sub-presentation of $\mathbf{P}=\left\langle x_{1}, \ldots, x_{n} \mid r_{1}, \ldots, r_{m}\right\rangle$ is a presentation $\left\langle y_{1}, \ldots, y_{n^{\prime}} \mid s_{1}, \ldots, s_{m^{\prime}}\right\rangle$ with each $y_{i} \in\left\{x_{1}, \ldots, x_{n}\right\}$ and each $s_{i} \in\left\{r_{1}, \ldots, r_{m}\right\}$ is only a word of $y_{1}, \ldots, y_{n^{\prime}}$.

Lemma 4.5. Suppose that $\mathbf{P}^{\prime}=\left\langle y_{1}, \ldots, y_{n^{\prime}} \mid s_{1}, \ldots, s_{m^{\prime}}\right\rangle$ is a sub-presentation of $\mathbf{P}=\left\langle x_{1}, \ldots, x_{n} \mid r_{1}, \ldots, r_{m}\right\rangle$ of a group $G_{\mathbf{P}}$. If $\mathbf{P}^{\prime \prime}$ is another finite presentation of $G_{\mathbf{P}^{\prime}}$, then one can obtain a presentation of $G_{\mathbf{P}}$ from $\mathbf{P}^{\prime \prime}$ by adding $n-n^{\prime}$ generators and $m-m^{\prime}$ relations. In particular, if $\mathbf{P}$ realizes $\mu_{2}^{g}\left(G_{\mathbf{P}}\right)$, then $\mathbf{P}^{\prime}$ realizes $\mu_{2}^{g}\left(G_{\mathbf{P}^{\prime}}\right)$.

Proof. Re-indexing and re-naming if necessary, we assume that

$$
y_{1}=x_{1}, \ldots, y_{n^{\prime}}=x_{n^{\prime}}, n^{\prime} \leqslant n
$$

and

$$
s_{1}=r_{1}, \ldots, s_{m^{\prime}}=r_{m^{\prime}}, m^{\prime} \leqslant m .
$$

It is clear that the words corresponding to $s_{1}, \ldots, s_{m^{\prime}}$ do not involve $x_{n^{\prime}+1}, \ldots, x_{n}$. If

$$
\mathbf{P}^{\prime \prime}=\left\langle y_{1}^{\prime}, \ldots, y_{u}^{\prime} \mid s_{1}^{\prime}, \ldots, s_{v}^{\prime}\right\rangle
$$

is another presentation of $G_{\mathbf{P}^{\prime}}$, we form a group $G^{\prime \prime}$ with the presentation

$$
\left\langle y_{1}^{\prime}, \ldots, y_{u}^{\prime}, x_{n^{\prime}+1}, \ldots, x_{n} \mid s_{1}^{\prime}, \ldots, s_{v}^{\prime}\right\rangle
$$

by adding $n-n^{\prime}$ free generators to $\mathbf{P}^{\prime \prime}$. For each $1 \leqslant i \leqslant n^{\prime}$, the letter $x_{i}$, viewed as an element in $G_{\mathbf{P}^{\prime}}$, has a lifting $w_{i}$ in the free group $\left\langle y_{1}^{\prime}, \ldots, y_{u}^{\prime}\right\rangle$. In other words, we choose $w_{i}$ on the generators $y_{1}^{\prime}, \ldots, y_{u}^{\prime}$ such that the bijection $x_{i} \mapsto w_{i}, 1 \leqslant i \leqslant n^{\prime}$ induces an isomorphism $G_{\mathbf{P}^{\prime}} \rightarrow G_{\mathbf{P}^{\prime \prime}}$.

For each $1 \leqslant i \leqslant n$, define the word $\omega_{i}$ of $\left\{y_{1}^{\prime}, \ldots, y_{u}^{\prime}, x_{n^{\prime}+1}, \ldots, x_{n}\right\}$ as

$$
\omega_{i}= \begin{cases}w_{i}, & 1 \leqslant i \leqslant n^{\prime} \\ x_{i}, & n^{\prime}<i \leqslant n\end{cases}
$$

Denote by $\phi$ the bijection

$$
\phi:\left\{x_{1}, \ldots, x_{n}\right\} \rightarrow\left\{\omega_{1}, \ldots, \omega_{n}\right\}
$$

given by $x_{i} \mapsto \omega_{i}$. For each $m^{\prime}<i \leqslant m$, write $r_{i}=\Pi_{j=1}^{k_{i}} x_{i j}$ as a reduced word of $\left\{x_{1}, \ldots, x_{n}\right\}$, where $x_{i j} \in\left\{x_{1}^{ \pm}, \ldots, x_{n}^{ \pm}\right\}$. Let $r_{i}^{\prime}=\Pi_{j=1}^{k_{i}} \phi\left(x_{i j}\right)$ be the corresponding word of

$$
\left\{y_{1}^{\prime}, \ldots, y_{u}^{\prime}, x_{n^{\prime}+1}, \ldots, x_{n}\right\} .
$$

Let $K$ be the normal subgroup of $G^{\prime \prime}$ normally generated by the $m-m^{\prime}$ elements $r_{m^{\prime}+1}^{\prime}, \ldots, r_{m}^{\prime}$. We obtain a short exact sequence of groups 


$$
1 \rightarrow K \rightarrow G^{\prime \prime} \rightarrow G_{\mathbf{P}} \rightarrow 1,
$$

where the third arrow is induced by the map $G_{\mathbf{P}^{\prime}} \rightarrow G_{\mathbf{P}}$ from the natural inclusions of generators and relators. From this exact sequence, one obtains the desired presentation

$$
\mathbf{P}_{0}=\left\langle y_{1}^{\prime}, \ldots, y_{u}^{\prime}, x_{n^{\prime}+1}, \ldots, x_{n} \mid s_{1}^{\prime}, \ldots, s_{v}^{\prime}, r_{m^{\prime}+1}^{\prime}, \ldots, r_{m}^{\prime}\right\rangle
$$

of $G_{\mathbf{P}}$.

Assume that $\mathbf{P}$ realizes $\mu_{2}^{g}\left(G_{\mathbf{P}}\right)$, while a sub-presentation $\mathbf{P}^{\prime}$ does not realize $\mu_{2}^{g}\left(G_{\mathbf{P}^{\prime}}\right)$. Suppose that $\mu_{2}^{g}\left(G_{\mathbf{P}^{\prime}}\right)$ is realized by a 2 -dimensional complex $X$, which gives a presentation $\mathbf{P}^{\prime \prime}$. We obtain a new presentation $\mathbf{P}_{0}$ of $G_{\mathbf{P}}$ by adding relators and generators to $\mathbf{P}^{\prime \prime}$. However,

$$
\chi\left(\mathbf{P}_{0}\right)=\chi\left(\mathbf{P}^{\prime \prime}\right)+m-m^{\prime}-\left(n-n^{\prime}\right)=\chi\left(\mathbf{P}^{\prime \prime}\right)-\chi\left(\mathbf{P}^{\prime}\right)+\chi(\mathbf{P})<\mu_{2}^{g}\left(G_{\mathbf{P}}\right) .
$$

This is a contradiction to the fact that $\mathbf{P}$ realizes $\mu_{2}^{g}\left(G_{\mathbf{P}}\right)$. Therefore, $\mathbf{P}^{\prime}$ realizes $\mu_{2}^{g}\left(G_{\mathbf{P}^{\prime}}\right)$.

Recall that a CW complex $X$ is aspherical if the universal cover $\tilde{X}$ is contractible. A famous conjecture of Whitehead says that any subcomplex $Y$ of an aspherical 2dimensional complex $X$ is aspherical as well (for more details, see the survey article $[2])$. As an application of results proved above, we give an equivalent condition of the asphericity of $Y$, as follows.

Corollary 4.6. Suppose that $X$ is a finite aspherical 2-complex and $Y$ is a subcomplex of $X$. We have the following:

(i) The complex $Y$ realizes $\mu_{2}^{g}\left(\pi_{1}(Y)\right)$;

(ii) The complex $Y$ is aspherical if and only if the fundamental group $\pi_{1}(Y)$ has a finite classifying space $\mathrm{B} \pi_{1}(Y)$ of dimension at most 2.

Proof. Since $X$ is aspherical, it realizes $\mu_{2}^{g}\left(\pi_{1}(X)\right)$ by Theorem 4.3. Notice that $Y$ gives a presentation of $\pi_{1}(Y)$, which is a sub-presentation of the presentation given by $X$. Lemma 4.5 implies that $Y$ realizes $\mu_{2}^{g}\left(\pi_{1}(Y)\right)$. This proves part (i).

If $Y$ is aspherical, it is $\mathrm{B} \pi_{1}(Y)$ and hence is of dimension at most 2. Conversely, assume that $\pi_{1}(Y)$ has a finite classifying space $\mathrm{B} \pi_{1}(Y)$ of dimension at most 2 . By Theorem 4.3, all the $\left(\pi_{1}(Y), 2\right)$-complexes realizing $\mu_{2}^{g}\left(\pi_{1}(Y)\right)$ are homotopic to $\mathrm{B} \pi_{1}(Y)$. Therefore, $Y$ is aspherical by part (i).

Corollary 1.3 is Corollary 4.6 (ii).

\section{Acknowledgments}

The second author is supported by Jiangsu Natural Science Foundation (No. BK20140402) and NSFC (Nos. 11501459, 11771345, 11771022).

\section{References}

[1] G. Baumslag, A.G. Myasnikov, and V. Shpilrain, Open problems in combinatorial group theory, second edition, in: Combinatorial and Geometric Group Theory (New York, 2000/Hoboken, NJ, 2001), volume 296 of Contemp. Math., pages 1-38. Amer. Math. Soc., Providence, RI, 2002. 
[2] W.A. Bogley, J.H.C. Whitehead's asphericity question, in: Two dimensional Homotopy and Combinatorial Group Theory, eds. C. Hog-Angeloni, A. Sieradski and W. Metzler, volume 197 of London Math. Soc. Lecture Note Ser., pages 309-334, Cambridge Univ Press, 1993.

[3] W. Browning, Homotopy types of certain finite $C W$-complexes with finite fundamental group, PhD thesis, Cornell University, 1978.

[4] T. Edwards, Generalized Swan modules and the D(2) problem, Algebr. Geom. Topol., 6(2006): 71-89.

[5] I. Hambleton, Two remarks on Wall's D2 problem, arXiv:1708.08532.

[6] J. Harlander and J. Jensen, On the homotopy type of CW complexes with aspherical fundamental group, Topology Appl., 153(2006): 3000-3006.

[7] X. Jin, Y. Su, and L. Yu, Homology roses and the D(2)-problem, Sci. China Math., 58(2015): 1753-1770.

[8] F.E.A. Johnson, Stable Modules and the D(2)-Problem, volume 301 of London Math. Soc. Lecture Note Ser., Cambridge University Press, 2003.

[9] F.E.A. Johnson, Syzygies and Homotopy Theory, volume 17 of Algebr. Appl., Springer-Verlag, 2012.

[10] C. Hog-Angeloni, W. Metzler, A.J. Sieradski (eds.), Two-Dimensional Homotopy and Combinatorial Group Theory, Cambridge University Press, 1993.

[11] J.C. Lennox and J. Wiegold, Generators and killers for direct and free products, Arch. Math. (Basel), 34(1980): 296-300.

[12] W. Mannan, The D(2) property for $D_{8}$, Algebraic \& Geom. Topol., 7(2007): $517-528$.

[13] W. Mannan, Quillen's plus-construction and the D(2) problem, Algebr. Geom. Topol., 9(2009): 1399-1411.

[14] W. Mannan, Realizing algebraic 2-complexes by cell complexes, Math. Proc. Cambridge Philos. Soc., 146(2009): 671-673.

[15] V.D. Mazurov and E.I. Khukhro (eds.), The Kourovka notebook, Russian Academy of Sciences Siberian Division Institute of Mathematics, Novosibirsk, augmented edition, 1999. Unsolved problems in group theory.

[16] R.G. Swan, Minimal resolutions for finite groups, Topology 4(1965): 193-208.

[17] C.T.C. Wall, Finiteness conditions for CW complexes, Ann. of Math. 81(1965): $55-69$.

[18] S. Ye, A unified approach to the plus-construction, Bousfield localization, Moore spaces and zero-in-the-spectrum examples, Israel J. Math., 192(2012): 699-717.

Feng Ji JIFENG@ntu.edu.sg

Infinitus, Nanyang Technological University, Singapore, 639798

Shengkui Ye Shengkui.Ye@xjtlu.edu.cn

Department of Mathematical Sciences, Xi'an Jiaotong-Liverpool University, 111 Ren Ai Road, Suzhou, Jiangsu, China 215123 